\title{
Vicia faba hull: A novel source of fibre, and a functional food with antidiabetic properties
}

\author{
Viren Ranawana $^{1}$, Gordon McDougall ${ }^{2}$, Nicholas Hayward ${ }^{1}$ and Vassilios Raikos ${ }^{1}$ \\ ${ }^{1}$ University of Aberdeen, Aberdeen, United Kingdom and \\ ${ }^{2}$ The James Hutton Institute, Dundee, United Kingdom
}

\section{Abstract}

Combinable broad beans (Vicia faba) is a major agricultural crop in Europe with annual production around 1.5 million mt. During its milling the seed testa (hull) is removed and is a significant underutilised secondary product. The aim of this study was to examine the nutritional and functional properties of broad bean hull (BBH), specifically for blood glucose control and as a source of fibre for humans.

The proximate composition of BBH was assessed using standard AOAC methods and the phytochemical profile using LC-MS ${ }^{(1)}$. BBH extracts were characterised for total polyphenol content, antioxidant potential (FRAP, TEAC) ${ }^{(2)}$, and effects on $\alpha$-amylase (AA) and $\alpha$-glucosidase (AG) enzyme activity ${ }^{(3)}$. Bread containing BBH was developed and assessed for glycaemic potential using a standardised in vitro gastro-intestinal digestion model ${ }^{(4)}$. Wheat bran and acarbose were used as comparators.

BBH showed a comparable proximate composition to wheat bran but with higher fibre ( $49 \mathrm{vs} 43 \mathrm{~g} / 100 \mathrm{~g}$ ), and lower carbohydrate $(19 \mathrm{vs} 23 \mathrm{~g} / 100 \mathrm{~g})$ and fat contents $(1.3 \mathrm{vs} 5 \mathrm{~g} / 100 \mathrm{~g})$. LC-MS profiling showed that proanthocyanidins were the predominant phytochemicals in the hull with some minor compounds including flavonols. Total polyphenol content and antioxidant potential (FRAP, TEAC) of BBH was significantly higher than in wheat bran (160.1 vs $12.3 \mu \mathrm{g}$ Gallic acid/mL, 3877.9 vs $75.5 \mu \mathrm{M} \mathrm{Fe}$ Sulphate, and 0.50 vs $0.19 \mathrm{mM}$ Trolox Eq respectively; $\mathrm{P}<0.001$ ). Compared to acarbose $\mathrm{BBH}$ showed a significantly higher $\mathrm{IC}_{50}$ for $\alpha$-amylase inhibition (76.6 vs $4.2 \mu \mathrm{g} / \mathrm{mL}$ ), and a significantly lower value for $\alpha$-glucosidase inhibition $(2.7 \mathrm{vs} 2175.8 \mu \mathrm{g} / \mathrm{mL})(\mathrm{P}<$ 0.001). The addition of $\mathrm{BBH}$ significantly reduced starch digestion in bread during the oral, gastric phases and at 20 and $60 \mathrm{~min}$ of intestinal digestion $(\mathrm{P}<0.005)$.

Broad bean hull is a good source of non-soluble fibre with potential for increasing fibre contents in foods by up to $15 \mathrm{~g} / 100 \mathrm{~g}$. Compared to similar material it is a richer source of polyphenols and antioxidants, retards both $\alpha$-amylase and $\alpha$-glucosidase activity and shows potential for reducing the glycaemic potential of foods.

The work was funded by the Rural and Environment Science and Analytical Services Division of the Scottish government (RESAS).

\section{Conflict of Interest}

There is no conflict of interest

\section{References}

1. Brown E, Gill C et al. (2016) Journal of Berry Research 6(1), 13-23.

2. Ranawana V, Campbell F et al. (2016) Foods 5(3), 62-76.

3. Pantidos N, Boath et al. (2014) Journal of Functional Foods 10, 201-209.

4. Minekus M, Alminger M et al. (2014) Food \& function 5(6), 1113-1124. 